transported in dry contains for the detection of STI with 'Multiplex Real-time PCR' assays. The objective of this study was to validate the performance of urine in UriSponge ${ }^{\mathrm{TM}}$ for the detection of STI with the Seegene Anyplex ${ }^{\text {TM }}$ II-STI-7 assay

Methods Urine collected with UriSponge ${ }^{\text {TM }}$ from 2991 patients attending a STD-clinic were used in this study. A first void urine was collected in a sterile-cap, the UriSponge ${ }^{\mathrm{TM}}$ sponges were immersed into the urine until saturated and stored back in its own tubes. Prior testing, UriSponge ${ }^{\mathrm{TM}}$ samples were centrifuged at 2500-RPM for $5 \mathrm{~min}$, de-capped the tubes and discarded the sponges, vortexed and loaded the urine tubes on the Nimbus for nucleic acids extraction and amplified with the Anyplex II STI-7-assay for the detection of Chlamydia trachomatis (CT, Neisseria gonorrhoeae (NG), Trichomonas vaginalis (TV), Mycoplasma genitalium (MG), Mycoplasma hominis (MH),Ureaplasma urealyticum (UU), and Ureaplasma Parvum (UP).

Results In the 2991 UriSponge $^{\mathrm{TM}}$ samples tested, were detected 267 (8.02\%) CT, 220 (7.35\% NG, 59 (1.97\%) TV, 129 (4.31\%) MG, 232 (7.75\%) MH, 430 (14.5\%) UU and $640(21.4 \%)$ UP positive. The positivity rate of each STI was comparable to data obtained from a previous study comparing urine samples stored in sterile containers and UriSponge ${ }^{\mathrm{TM}}$. Samples processing on the Nimbus facilitated testing workflow. Conclusion Good performance was obtained with urine collected and transported with UriSponge ${ }^{\mathrm{TM}}$ for the detection of STI with the Anyplex TM II STI-7-assay. UriSponge ${ }^{\mathrm{TM}}$ is an optimal device for urine collection for STI screening, eliminating the use of large leaking urine containers and are supporting culture for NG AST.

Disclosure No significant relationships.

\section{P021 USE OF NON-FDA CLEARED ASSAYS FOR DETECTION OF STIS IN CHILDREN BEING EVALUATED FOR SUSPECTED SEXUAL ABUSE}

${ }^{1}$ Margaret Hammerschlag* ${ }^{*}{ }^{2}$ Amanda Brownell, ${ }^{2}$ Robert Shapiro. ${ }^{1}$ State University of New York Downstate Medical Center, Pediatrics, Brooklyn, USA; ${ }^{2}$ Cincinnati Children's Hospital, Mayerson Center for Safe and Healthy Children, Cincinnati, USA

\subsection{6/sextrans-2019-sti.230}

Background Although NAAT testing is the gold-standard for C. trachomatis (Ct) and GC in adults, limited data exists for children. Although there are now 7 FDA cleared $\mathrm{Ct} / \mathrm{GC}$ NAATs in the US, assays that are not FDA cleared have been proliferating.

Methods We present 3 instances where non approved assays were used in children being evaluated for suspected sexual abuse.

Results The tests were Vaginitis Plus NuSwab ${ }^{\circledR}$ (LabCorp, North Carolina) which tests for BV, Candida species, Ct, GC, and T. vaginalis (Tv), Diatherix Laboratories NAAT for GC, $\mathrm{Ct}$ and Tv (Huntsville, Alabama) and 'Leukorrhea Panel' (MDLabs, NJ), R-T PCR for Tv, GC and Ct. These tests are not FDA-cleared. There are no studies on the performance of these assays in men or women in the peer-reviewed literature including sensitivity, specificity (including cross reactivity with other bacteria) and predictive values for any anatomic site. MDLabs also claimed that their assay did 'reflex' antibiotic suseptibility testing for GC and Ct. A 11 yo girl with a positive $\mathrm{NuSwab}$ for $\mathrm{Ct}$ subsequently tested negative by TMA. A 2 yo boy tested positive for GC by Diatherix in a rectal specimen, which was discarded after 7 days. The Diatherix manual specifically stated that the test should not be used for rectal specimens. Two siblings tested positive in throat for GC by the 'Leukorrhea Panel' after disclosure of oral penile contact, but father was negative for GC. Specimens were not retained for confirmatory testing.

Conclusion Because of low STI disease prevalence in children (<3\%), low test specificity calculates to an unacceptably low positive predictive value. None of these labs retained the specimens for confirmatory testing, a major departure from CDC recommendations. Use of NAATs that have not been cleared by any regulatory agency without reliable test specificity data can have criminal and juvenile protection implications when used in children.

Disclosure No significant relationships.

\section{P022 ANTENATAL TESTING FOR CURABLE STIS COMPARED TO SYNDROMIC MANAGEMENT IN BOTSWANA: A COST-EFFECTIVENESS STUDY}

${ }^{1}$ Adriane Wynn*, ${ }^{2}$ Corrina Moucheraud, ${ }^{3}$ Chelsea Morroni, ${ }^{4}$ Doreen Ramogola-Masire, ${ }^{5}$ Jeffrey Klausner, ${ }^{6}$ Arleen Leibowitz. ${ }^{1}$ UCSD, Medicine, La Jolla, USA; ${ }^{2}$ UCLA, Fielding School of Public Health, Los Angeles, USA; ${ }^{3}$ Botswana-UPenn Partnership, Gaborone, Botswana; ${ }^{4}$ University of Botswana, Gaborone, Botswana; ${ }^{5}$ UCLA - David Geffen School of Medicine, Infectious Diseases, Los Angeles, USA; ${ }^{6}$ UCLA, Luskin School of Public Affairs, Los Angeles, USA

\subsection{6/sextrans-2019-sti.231}

Background Chlamydia trachomatis (CT) and Neisseria gonorrhoeae (NG) are sexually transmitted infections (STIs) associated with adverse outcomes, including low birth weight (LBW). Most countries don't test pregnant women and use syndromic management, which misses asymptomatic infections. This study used a decision model to assess the cost-effectiveness of testing pregnant women for CT and NG using the GeneXpert compared to syndromic management in Botswana. Methods Using costs and implementation data from a previous study and outcome data from the literature, we modelled the short-term incremental costs and neonatal outcomes of both scenarios from a health services prospective. For the base case, we assumed CT and NG prevalence rates were $7.8 \%$ and $1.3 \%$, and the probabilities of LBW associated with maternal CT was $5 \%$ and NG was 58\%. Probabilities of death and disability weights came from the World Health Organization.

Results Offering CT and NG testing to approximately 50,000 antenatal patients in Botswana had a one-year cost of $\$ 925,804$ more than syndromic management. Testing was associated with 4,322 more cured maternal infections, and 612 more cases of LBW infants averted, which is $\$ 1,513$ per LBW infant averted and $\$ 320$ per disability adjusted life year (DALY) averted. The incremental cost-effectiveness ratios were most sensitive to the assumed prevalence of CT and NG, probability of LBW, disability weight, treatment uptake, and capital costs.

Conclusion Testing for CT and NG infections is more costly than syndromic management. However, testing is estimated to prevent LBW infants and DALYs. CT/NG testing scale-up is cost-effective if policy-makers' willingness to pay is informed by the WHO 1 Gross Domestic Product/capita threshold (\$7,596/DALY averted in Botswana). While the costs of testing may be high for countries with constrained budgets in Southern Africa, reductions in antenatal STIs may help address the important global goal of preventing LBW.

Disclosure No significant relationships. 